\title{
In Vivo Transfection of Naked DNA into Xenopus Tadpole Tail Muscle
}

\author{
Lindsey Marshall, ${ }^{1}$ Fabrice Girardot, ${ }^{1}$ Barbara A. Demeneix, ${ }^{1}$ and Laurent Coen ${ }^{1,2}$ \\ ${ }^{1}$ Evolution des Régulations Endocriniennes, Département RDDM, CNRS UMR 7221, MNHN, Sorbonne \\ Université, Paris, France
}

In vivo gene transfer systems are important to study foreign gene expression and promoter regulation in an organism, with the benefit of exploring this in an integrated environment. Direct injection of plasmids encoding exogenous promoters and genes into muscle has numerous advantages: the protocol is easy, efficient, and shows time-persistent plasmid expression in transfected muscular cells. After injecting naked-DNA plasmids into tadpole tail muscle, transgene expression is strong, reproducible, and correlates with the amount of DNA injected. Moreover, expression is stable as long as the tadpoles remain, or are maintained, in premetamorphic stages. By directly expressing genes and regulated promoters in Xenopus tadpole muscle in vivo, one can exploit the powerful experimental advantages of gene transfer systems in an intact, physiologically normal animal.

\section{MATERIALS}

It is essential that you consult the appropriate Material Safety Data Sheets and your institution's Environmental Health and Safety Office for proper handling of equipment and hazardous materials used in this protocol.

RECIPES: Please see the end of this protocol for recipes indicated by $<R>$. Additional recipes can be found online at http://cshprotocols.cshlp.org/site/recipes.

Reagents

\section{DNA injection mixture}

Dilute ultrapure stock solution(s) of plasmid(s) (obtained using a standard Maxiprep kit) to a final concentration of $0.1-0.5 \mathrm{~g} / \mathrm{L}$ in $0.075 \mathrm{M} \mathrm{NaCl}$ with $1 \times$ Fast Green solution. Prepare the mixture on the day of injections, adapting the volume to the number of tadpoles to inject. Maintain at room temperature until use.

Fast Green stock solution (10X)

Dissolve $1 \mathrm{mg}$ of Fast Green FCF dye (Sigma-Aldrich, F7252) in $1 \mathrm{~mL}$ of sterile, double-distilled water. Store at $-20^{\circ} \mathrm{C}$. Dilute to $1 \times$ before use.

MS222 solution $(0.1 \%)<\mathrm{R}>$

Also known as tricaine methanesulfonate or ethyl 3-aminobenzoate methanesulfonate, MS222 is the main anesthetic used for aquatic models. MS222 can cause skin, eye, and even respiratory irritation. Use personal protective equipment as required.

Water, dechlorinated, $21^{\circ} \mathrm{C}$

\footnotetext{
${ }^{2}$ Correspondence: laurent.coen@mnhn.fr From the Xenopus collection, edited by Hazel L. Sive.

(c) 2017 Cold Spring Harbor Laboratory Press

Cite this protocol as Cold Spring Harb Protoc; doi:10.1101/pdb.prot099366
} 


\section{Equipment}

Water, sterile, double-distilled

Xenopus laevis tadpoles, premetamorphic stages NF55-NF57 (Nieuwkoop and Faber 1994) Injections can be performed on younger tadpoles, but because of their smaller size and thinner tail muscles the success of the experiment is less assured. Likewise, older animals can be used, but the experiment must be designed such that it can be completed before the peak of metamorphosis when tail regression and muscle apoptosis occur.

Acupuncture needle, 0.25-mm diameter

Electrode puller (e.g., Narishige Group, Model Pb-7 or equivalent)

Gauze

Injection holder set for 1.0-mm microcapillary tubing (Narishige Group, Model IM-H1)

The set consists of an HI-7 injection holder, CT-1 PTFE tubing and Cl-1 tube connector.

Microcapillary tubing, glass, 1.0 mm (Drummond Scientific, Cat. \#1-000-0300)

Microdissection forceps

Microinjector (Narishige Group, Model IM-6)

Micromanipulator (Narishige Group, Model M-152)

This is used to precisely position the injection holder on three axes.

P2 pipette (Gilson or equivalent)

Paraffin mold

Pipette tips, sterile, $10-\mu \mathrm{L}$ (to fit a $\mathrm{P} 2$ pipette)

Steel sheet, galvanized, $35 \mathrm{~cm} \times 45 \mathrm{~cm} \times \geq 2 \mathrm{~mm}$

Stereomicroscope and light source

Syringe, $50-\mathrm{mL}$

Tanks

One tank is used for MS222; three for dechlorinated water (pre- and post-anesthesia, and post-injection).

Teflon tubing, 1-mm i.d.

\section{Injection System Setup}

1. Assemble the injection system (Fig. 1A):

i. Attach the micromanipulator and the microinjector to the steel sheet.

ii. Support the injection holder set with the micromanipulator.

iii. Connect the microinjector to the injection holder set.

2. Using a syringe, fill the injection system with sterile double-distilled water through the inlet at the top of the microinjector (Fig. 1A).

3. Bleed any air bubbles from the injection system.

4. Use the electrode puller to prepare needles from glass microcapillary tubes. Settings for the Narishige model Pb-7 correspond to: No. 1 Heater $=60 ;$ No. 2 Heater $=70$.

5. Use microdissection forceps to bevel the elongated needle extremity such that length of the elongated end is $\sim 2-2.5 \mathrm{~mm}$ and the outer diameter of the needle tip is $\sim 10-15 \mu \mathrm{m}$.

This can be verified using a graduated stage micrometer measurement tool.

6. Insert the needle in the injection holder containing a small piece of Teflon tube that will be tightened around the needle with the outer screw. 
L. Marshall et al.
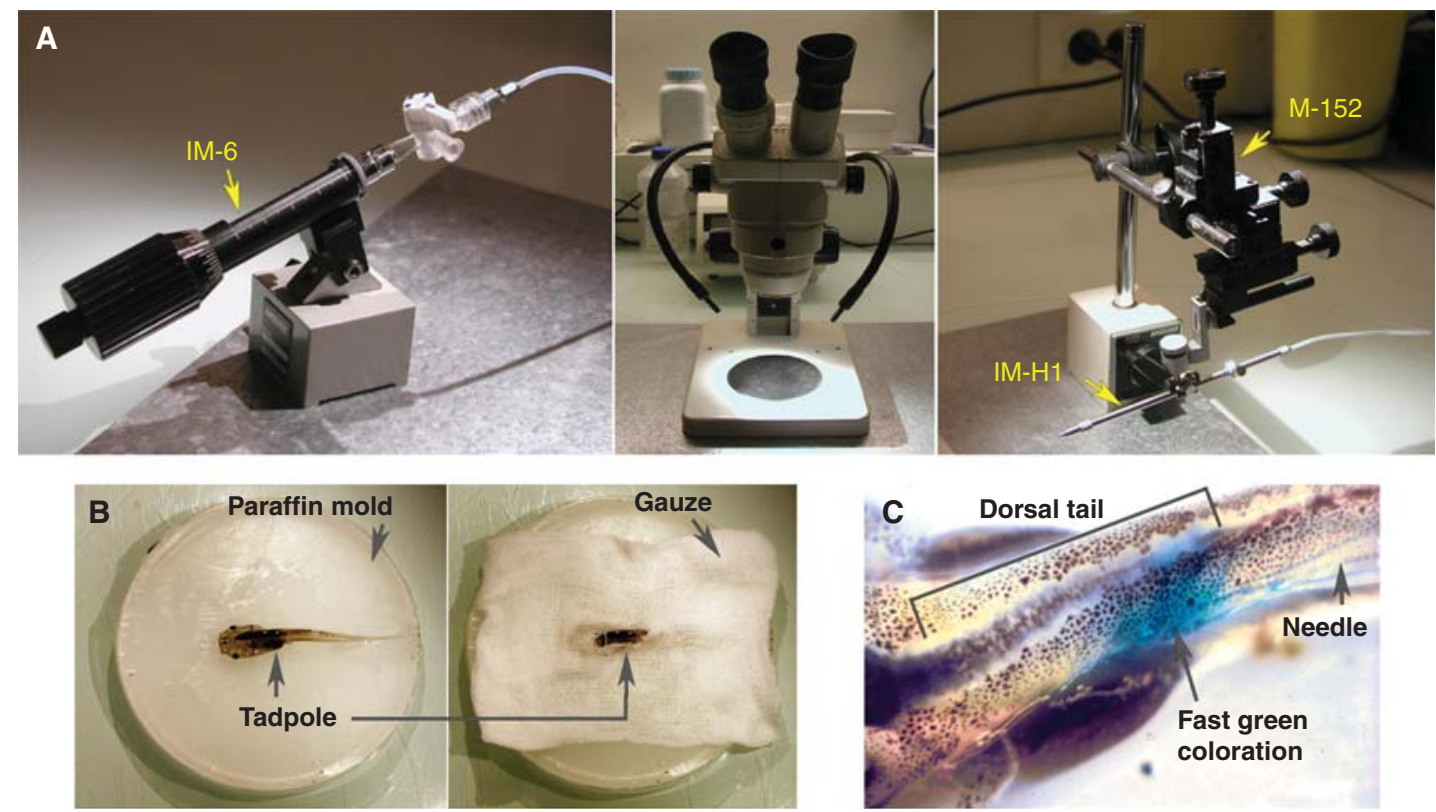

D CMV-GFP injection
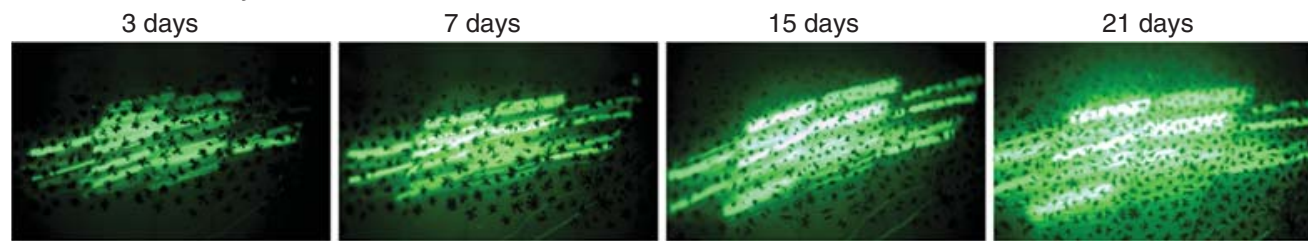

E CMV-LUC injection

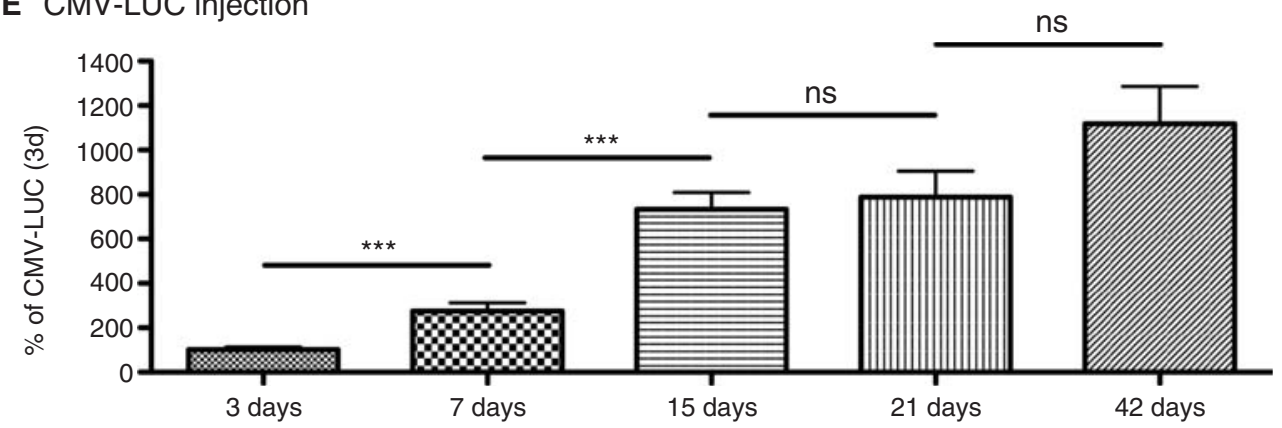

FIGURE 1. Workstation setup, tadpole positioning and injection, and potential analysis. Key steps in the protocol. $(A)$ The workstation includes a microinjector (IM-6; left), microscope and light (center) and injection holder set (IM-H1) supported by a micromanipulator (M-152; right). (B) Tadpole positioned on a paraffin mold (left) and covered with a moistened gauze that has an opening exposing the tail (right). (C) Fast Green makes the mixture injected into the muscle visible. $(D)$ Increasing GFP fluorescence observed on a live animal injected with a mixture containing a cytomegalovirus-green fluorescent protein (CMV-GFP) plasmid (200 ng/ $\mu \mathrm{L}$ ) at various times post-injection. (E) Quantification of luciferase (LUC) activity in extracts from muscles injected with a mixture containing a CMV-LUC plasmid (200 ng/ $\mu \mathrm{L}, 10$ animals per time point), showing a maximum activity detected after $15 \mathrm{~d}$ post-injection. ( $D$ and $E$ adapted from Vivien et al. [2012].)

Tadpole Preparation

7. Anesthetize a NF55-NF57 stage tadpole by immersing it in freshly prepared MS222 solution.

The tadpole should stop moving in $\sim 15-30$ sec.

8. Transfer the anesthetized tadpole to a tank of clean dechlorinated water to rinse off the MS222.

9. Gently place the tadpole ventral-side down on the paraffin mold. Cover with a piece of moist gauze into which an opening has been cut that allows access to the tail muscle (Fig. 1B). 
Filling the Needle

10. Adjust the needle in a slightly oblique position with the tip visible under the microscope.

11. Aspirate $1 \mu \mathrm{L}$ of DNA injection mixture into a $10-\mu \mathrm{L}$ pipette tip. Gently remove the tip from the pipette. Using one's thumb, press the large end of the tip to force the liquid back toward the end.

12. While viewing under the microscope, approach the tip with the needle's end until it is in the liquid inside the pipette tip. Steadily holding that position, gradually move the injector dial counter-clockwise to load $1 \mu \mathrm{L}$ of the injection mixture into the needle (see online Movie 1 at cshprotocols.cshlp.org).

Control the flow carefully. Reserve an air space between the injection system water and the solution to prevent mixing.

Tadpole Injection (see Movie 2 at cshprotocols.cshlp.org)

13. Position the prepared tadpole under the microscope such that both the tadpole tail muscle and the filled needle are visible.

14. Select a suitable visual cue (e.g., a melanocyte) located 5-6 myomeres from the head and positioned at the top of the selected myomere. Pierce the skin at the selected point using the acupuncture needle.

Wipe the needle clean with $70 \%$ ethanol between each injected animal.

15. Rotate the mold slightly. Position the micromanipulator to guide the needle into the tadpole muscle through the pierced hole.

16. With the needle's end now inside the myomere, rotate the mold back to its original position such that the tail muscle parallels the needle.

17. Drive the needle forward through three myomeres. Withdraw slightly.

Resistance occurs each time the needle passes through a myomere, allowing them to be counted.

18. Slowly inject $1 \mu \mathrm{L}$ of mixture by rotating the injector dial clockwise. Wait a few seconds.

The mixture often diffuses into two to three myomeres.

See Troubleshooting.

19. Slowly withdraw the needle from the tadpole, controlling the pressure to prevent injection of air bubbles.

The mixture injected into the myomeres can be observed by the Fast Green coloration for a few minutes (Fig. 1C).

See Troubleshooting.

20. Transfer the injected tadpole to a new tank of clean dechlorinated water and allow it to recover. It should start to swim within $\sim 5$ min.

See Troubleshooting.

21. Maintain the tadpoles in dechlorinated water. Do not feed the tadpoles for $24 \mathrm{~h}$ following the injection, then feed every $2 \mathrm{~d}$ until the end of the experiment.

22. Depending on the reporter plasmid used, injected tadpoles can be monitored differently. For instance, fluorescent markers (e.g., GFP) can be monitored on living tadpoles (Fig. 1D), or luciferase activity can be quantified in extracted muscle tissue after tadpole euthanasia (Fig. 1E).

Problem (Step 18): The needle appears to be clogged.

Solution: Debris or muscle tissue can obstruct the needle tip. Slightly withdraw the needle and try again. 
L. Marshall et al.

Problem (Steps 18 and 19): The liquid is released too fast in the muscle. Air bubbles can be seen in the tissue.

Solution: Air injected into the tadpole can lead to impaired muscle integrity and transfection efficiency. Repeat the injection with a new tadpole.

Problem (Step 19): No Fast Green coloration is seen in muscle immediately after injection.

Solution: No staining in muscle indicates that the mixture was not injected properly, possibly by reaching too far in the general cavity. Repeat the injection with a new tadpole.

Problem (Step 20): Tadpole does not recover following injection.

Solution: In general we do not observe mortality following this procedure. However, possibilities include animal left too long in MS222 or out the water. Repeat the injection with a new tadpole.

\section{DISCUSSION}

Among nonviral techniques, direct injection of plasmids encoding exogenous promoters and genes into muscle was first established in mice (Wolff et al. 1990). This method was adapted and applied to Xenopus laevis in our laboratory (de Luze et al. 1993). In vivo somatic gene transfer in Xenopus tadpole muscle is particularly relevant for studying biological processes in which the integrated context is especially important. For instance, this technique has been used to study physiological regulations by thyroid hormone during amphibian metamorphosis (Sachs et al. 1996, 1998; Nakajima and Yaoita 2003). Testing for endocrine-disrupting effects of increasing numbers of substances present in the environment also benefits from this technique, as the in vivo context integrates all possible targets of the compounds and their metabolites resulting in higher specificity (Turque et al. 2005; Punzon et al. 2013). We recently used this technique to show the possibility to induce cell reprogramming in vivo with a high efficiency. We showed that Xenopus differentiated skeletal muscle fibers could be reprogrammed to pluripotency after transfecting the Yamanaka factors (mouse Oct4, Sox2 and Klf4). This was the first time that induced reprogramming was reported to be feasible in vivo and that mammalian factors were shown to be able to reprogram nonmammalian cells (Vivien et al. 2012); others have confirmed these observations (Abad et al. 2013; Rosselló et al. 2013). This safe and inexpensive technique has been adapted to fish (Hansen et al. 1991) including zebrafish (L. Coen, unpublished data), Xenopus tropicalis (Rowe et al. 2002), and used in various species to develop immunization protocols (Donnelly et al. 1995; Lewis and Babiuk 1999; Pachuk et al. 2000).

\section{MS222 Solution (0.1\%)}

MS222 (Sigma-Aldrich, A5040)

Sodium bicarbonate (Sigma-Aldrich, S5761) $1 \mathrm{~g}$

Water, dechlorinated

$1 \mathrm{~g}$

Protect from light. Store for up to $2 \mathrm{wk}$ at room temperature.

\section{REFERENCES}

Abad M, Mosteiro L, Pantoja C, Cañamero M, Rayon T, Ors I, Graña O, Megías D, Domínguez O, Martínez D, et al.. 2013. Reprogramming in vivo produces teratomas and iPS cells with totipotency features. Nature 502: $340-345$.

de Luze A, Sachs L, Demeneix B. 1993. Thyroid hormone-dependent transcriptional regulation of exogenous genes transferred into Xenopus tadpole muscle in vivo. Proc Natl Acad Sci 90: 7322-7326.
Donnelly JJ, Ulmer JB, Liu MA. 1995. Protective efficacy of intramuscular immunization with naked DNA. Ann N Y Acad Sci 772: 40-46.

Hansen E, Fernandes K, Goldspink G, Butterworth P, Umeda PK, Chang KC. 1991. Strong expression of foreign genes following direct injection into fish muscle. FEBS Lett 290: 73-76.

Lewis PJ, Babiuk LA. 1999. DNA vaccines: A review. Adv Virus Res 54: 129-188. 
Nakajima K, Yaoita Y. 2003. Dual mechanisms governing muscle cell death in tadpole tail during amphibian metamorphosis. Dev Dyn 227: 246-255.

Nieuwkoop PD, Faber J (eds.). 1994. Normal table of Xenopus laevis (Daudin): A systematical of chronological survey of the development from the fertilized egg till the end of metamorphosis. Garland Publishing, New York.

Pachuk CJ, McCallus DE, Weiner DB, Satishchandran C. 2000. DNA vaccines - Challenges in delivery. Curr Opin Molec Ther 2: 188-198.

Punzon I, Latapie V, Le Mével S, Hagneau A, Jolivet P, Palmier K, Fini JB, Demeneix BA. 2013. Towards a humanized PPAR $\gamma$ reporter system for in vivo screening of obesogens. Mol Cell Endocrinol 374: 1-9.

Rosselló RA, Chen C-C, Dai R, Howard JT, Hochgeschwender U, Jarvis ED. 2013. Mammalian genes induce partially reprogrammed pluripotent stem cells in non-mammalian vertebrate and invertebrate species. Elife 2: e00036.

Rowe I, Coen L, Le Blay K, Le Mével S, Demeneix BA. 2002. Autonomous regulation of muscle fibre fate during metamorphosis in Xenopus tropicalis. Dev Dyn 224: 381-390.
Sachs L, de Luze A, Lebrun JJ, Kelly PA, Demeneix BA. 1996. Use of heterologous DNA-based gene transfer to follow physiological, T3-dependent regulation of myosin heavy chain genes in Xenopus tadpoles. Endocrinology 137: 2191-2194.

Sachs LM, de Luze A, Demeneix BA. 1998. Studying amphibian metamorphosis by in vivo gene transfer. Ann N Y Acad Sci 839: 152-156.

Turque N, Palmier K, Le Mével S, Alliot C, Demeneix BA. 2005. A rapid, physiologic protocol for testing transcriptional effects of thyroid-disrupting agents in premetamorphic Xenopus tadpoles. Environ Health Perspect 113: 1588-1593

Vivien C, Scerbo P, Girardot F, Le Blay K, Demeneix BA, Coen L. 2012. Nonviral expression of mouse Oct4, Sox2, and Klf4 transcription factors efficiently reprograms tadpole muscle fibers in vivo. J Biol Chem 287: 7427-7435.

Wolff JA, Malone RW, Williams P, Chong W, Acsadi G, Jani A, Felgner PL. 1990. Direct gene transfer into mouse muscle in vivo. Science 247: $1465-1468$. 


\section{In Vivo Transfection of Naked DNA into Xenopus Tadpole Tail Muscle}

Lindsey Marshall, Fabrice Girardot, Barbara A. Demeneix and Laurent Coen

Cold Spring Harb Protoc; doi: 10.1101/pdb.prot099366

\begin{tabular}{|c|c|}
\hline $\begin{array}{r}\text { Email Alerting } \\
\text { Service }\end{array}$ & Receive free email alerts when new articles cite this article - click here. \\
\hline $\begin{array}{l}\text { Subject } \\
\text { Categories }\end{array}$ & $\begin{array}{l}\text { Browse articles on similar topics from Cold Spring Harbor Protocols. } \\
\text { Developmental Biology (728 articles) } \\
\text { DNA Delivery/Gene Transfer ( } 344 \text { articles) } \\
\text { DNA Delivery/Gene Transfer, general (341 articles) } \\
\text { Non-Viral Methods (226 articles) } \\
\text { Xenopus (210 articles) } \\
\text { Xenopus Transgenics (32 articles) }\end{array}$ \\
\hline
\end{tabular}

